\title{
Characterization of a novel secretory spherical body protein in Babesia orientalis and Babesia orientalis-infected erythrocytes
}

Jiaying Guo ${ }^{1,3}$, Muxiao Li ${ }^{1,3}$, Yali Sun ${ }^{1,3}$, Long Yu ${ }^{1,3}$, Pei He ${ }^{1,3}$, Zheng Nie ${ }^{1,3}$, Xueyan Zhan ${ }^{1,3}$, Yangnan Zhao ${ }^{1,3}$, Xiaoying Luo ${ }^{1,3}$, Sen Wang ${ }^{1,3}$, Siqi Aoyang ${ }^{1,3}$, Qin Liu ${ }^{1,3}$, Cuiqin Huang ${ }^{4}$, Lan He $\mathrm{H}^{1,2,3^{*}}$ and Junlong Zhao ${ }^{1,2,3}$

\begin{abstract}
Background: The spherical body, a membrane bound organelle localized in the apical organelle complex, is unique to Babesia and Theileria spp. The spherical body proteins (SBPs) secreted by spherical bodies include SBP1, SBP2, SBP3 and SBP4. Up to now, only SBP3 has been characterized in Babesia orientalis.

Methods: The BoSBP4 gene was amplified from CDNA and gDNA and cloned into the pGEX-6P-1 vector by homologous recombination, sequenced and analyzed by bioinformatics tools. The amino acid (aa) sequence of BoSBP4 was compared with that of Babesia bovis and Babesia bigemina as well as SBP3 of B. orientalis. The immunoreactivity was evaluated by incubating recombinant BoSBP4 (rBoSBP4) with the serum of $B$. orientalis-infected water buffalo. The native form of BoSBP4 was identified by incubating lysate of $B$. orientalis-infected water buffalo erythrocytes with the anti-rBoSBP4 mouse serum. The cellular localization of BoSBP4 was determined by indirect immunofluorescence assay.

Results: The full length of the BoSBP4 gene was estimated to be 945 bp without introns, encoding a 314 aa polypeptide with a predicted molecular weight of $37 \mathrm{kDa}$. The truncated recombinant protein was expressed from 70 to $945 \mathrm{bp}$ as a GST fusion protein with a practical molecular weight of $70 \mathrm{kDa}$. BoSBP4 shared a $40 \%$ and $30 \%$ identity with B. bovis and $B$. bigemina, respectively. Furthermore, it was 31\% identical to SBP3 of $B$. orientalis. BoSBP4 was identified in the lysate of $B$. orientalis-infected water buffalo erythrocytes with a molecular weight of $37 \mathrm{kDa}$, corresponding to the expected molecular mass of BoSBP4. The result of rBoSBP4 with positive serum revealed that BoSBP4 can elicit an immune response to $B$. orientalis-infected water buffalo. The cellular localization of BoSBP4 was detected to be adjacent to the merozoite nucleus in the intracellular phase, followed by the diffusion of the fluorescence of BoSBP4 into the cytoplasm of B. orientalisinfected erythrocytes as puncta-like specks and a gradual increase of the fluorescence.

Conclusions: In this study, SBP4 in B. orientalis was characterized for the first time. It may play a key role in interaction with the host cell by being secreted into the cytoplasm of the $B$. orientalis-infected erythrocytes to facilitate parasite growth and reproduction.
\end{abstract}

Keywords: Spherical body, Apical organelle complex, Babesia orientalis, Native form, Immunoreactivity, Cellular localization

\footnotetext{
* Correspondence: helan@mail.hzau.edu.cn

${ }^{1}$ State Key Laboratory of Agricultural Microbiology, College of Veterinary Medicine, Huazhong Agricultural University, Wuhan 430070, Hubei, China

${ }^{2}$ Key Laboratory of Animal Epidemical Disease and Infectious Zoonoses,

Ministry of Agriculture, Huazhong Agricultural University, Wuhan 430070,

Hubei, China

Full list of author information is available at the end of the article
}

(c) The Author(s). 2018 Open Access This article is distributed under the terms of the Creative Commons Attribution 4.0 International License (http://creativecommons.org/licenses/by/4.0/), which permits unrestricted use, distribution, and reproduction in any medium, provided you give appropriate credit to the original author(s) and the source, provide a link to the Creative Commons license, and indicate if changes were made. The Creative Commons Public Domain Dedication waiver (http://creativecommons.org/publicdomain/zero/1.0/) applies to the data made available in this article, unless otherwise stated. 


\section{Background}

Babesiosis is caused by species of the genus Babesia, and is one of the most epidemic infections distributed throughout the world [1-3]. Babesia spp. can cause different kinds of babesiosis in animals, and buffalo babesiosis greatly affects the cattle industry, leading to huge economic losses annually [3, 4]. It has been reported that several parasites can cause buffalo babesiosis, including Babesia bovis, Babesia bigemina and Babesia orientalis [4, 5]. Babesia orientalis was identified to be transmitted by Rhipicephalus haemaphysaloides in its original description in 1997 [6, 7]. Clinical symptoms for B. orientalis infection include anemia, fever, icterus and hemoglobinuria, and it can cause death in serious cases $[3,8]$.

To successfully invade the host cell, protozoan parasites need to depend on the parasite-derived proteins secreted by apical complex organelles $[9,10]$. The apical complex organelles consist of rhoptries, micronemes and dense granules [10]. Previous studies have shown that the proteins discharged by rhoptries and micronemes mainly participate in the initial attachment, invasion and the early stage of post-invasion processes [1114]. Dense granules are reported to release proteins into the parasitophorous vacuole (PV) shortly after invasion, which may play a key role in PV membrane modifications and is assumed to be associated with the nutrient acquisition. Likewise, the proteins secreted by dense granules are also translocated to the cytoplasmic side of infected red blood cell (iRBC) and participate in stabilizing spectrin tetramers [15-18]. However, in Babesia spp. and Theileria spp., spherical bodies are membranebound and localized to the apical organelles complex instead of dense granules $[9,19]$. The spherical body proteins (SBPs) secreted by spherical bodies are identified to belong to a family consisting of SBP1, SBP2, SBP3 and SBP4, which have been characterized in B. bovis [20-22]. Using immunoscreening, SBP1 was firstly identified from the genomic DNA (gDNA) of B. bovis merozoite with a molecular weight of $77 \mathrm{kDa}$ and located at the apex of the intraerythrocytic parasite [23]. In 1995, SBP1 was characterized to be localized to the spherical bodies by immunofluorescence and immunoelectron microscopy [19]. For SBP2, previous studies have reported a $225 \mathrm{kDa}$ protein from $B$. bovis, which is localized to the cytoplasmic side of the iRBC [22, 24]. SBP3 and SBP4 of $B$. bovis have been recently characterized and identified to localize to the cytoplasm of iRBC, rather than the cytoplasmic side of the $\operatorname{iRBC}[20,21,25]$. However, only the SBP3 of $B$. orientalis has been characterized so far in the merozoite to be discharged to the cytoplasm of iRBC [26].

Despite extensive research on SBPs and the identification of SBPs many years ago, their mechanisms and functions remain poorly understood. Furthermore, many studies have shown that antibodies against SBPs play significant roles in the protection of Babesia-infected cattle and are potential candidates for the development of vaccines [27-29]. Therefore, it is necessary to further investigate SBPs by evaluating their functions in interaction with the host cells.

\section{Methods}

Identification, cloning and sequencing of the BoSBP4 gene In order to find the BoSBP4 gene, the genome of $B$. orientalis (unpublished data) was screened using a Basic Local Alignment Search Tool (BLAST) (https://blast.ncbi.nlm.nih.gov/Blast.cgi), TBLASTN, with the reported SBP4 amino acid sequence of B. bovis (GenBank: AAL92106) and B. bigemina (GenBank: XP_012767973) as queries [3, $30,31]$. The gene with a significant similarity with SBP4 of $B$. bovis and B. bigemina was designated as BoSBP4 gene.

The following primers for cloning BoSBP4 gene were designed based on the in silico BLAST search result and the $B$. orientalis genome sequence: the forward primer (5'-ATG GTG GCT CTT TCC CTA CG-3') and the reverse primer (5'-TTA CTC AGT GGT GGT TTC GGT TTC-3').

The recombinant plasmid was constructed using the homologous recombination method. The following primers for cloning BoSBP4 gene and the pGEX-6p-1 vector were synthesized (Tianyi Huiyuan Biological Technology, Wuhan, China): the forward primer for cloning BoSBP4 gene (5'-TTC TGT TCC AGG GGC CCC TGG AGG AAG TTG TTG AGG AAC C-3') and the reverse primer (5'-GAT CGT CAG TCA GTC ACG AT GTT ACT CAG TGG TGG TTT CGG-3'); the forward primer for cloning pGEX-6p-1 vector (5'-CAT CGT GAC TGA CTG ACG ATC-3') and the reverse primer (5'-CAG GGG CCC CTG GAA CAG AA-3').

The complementary DNA (cDNA) and gDNA of $B$. orientalis were extracted and stored at $-80{ }^{\circ} \mathrm{C}$ as reported previously [26]. The entire length of the BoSBP4 gene was cloned from cDNA and gDNA. For construction of the recombination plasmid, the BoSBP4 gene and vector were amplified respectively from gDNA of $B$. orientalis and pGEX-6p-1 plasmid (Takara Biotechnology, Beijing, China) using corresponding homologous recombination primers as described above. The thermal cycling parameters for BoSBP4 gene included the initial denaturation at $95{ }^{\circ} \mathrm{C}$ for $5 \mathrm{~min}$; 33 cycles of denaturation at $94{ }^{\circ} \mathrm{C}$ for 30 $\mathrm{s}$, annealing at $55^{\circ} \mathrm{C}$ for $30 \mathrm{~s}$, extension at $72{ }^{\circ} \mathrm{C}$ for $3 \mathrm{~min}$; and a final extension of $10 \mathrm{~min}$ at $72{ }^{\circ} \mathrm{C}$. The vector cloning included the initial denaturation at $95{ }^{\circ} \mathrm{C}$ for $5 \mathrm{~min} ; 33$ cycles of denaturation at $94{ }^{\circ} \mathrm{C}$ for $30 \mathrm{~s}$, annealing at $55^{\circ} \mathrm{C}$ for $30 \mathrm{~s}$, extension at $72{ }^{\circ} \mathrm{C}$ for $6 \mathrm{~min}$; and a final extension of $10 \mathrm{~min}$ at $72{ }^{\circ} \mathrm{C}$.

The PCR products were electrophoresed using $2 \%$ ethidium bromide-stained agarose gel and purified using an 
EasyPure $^{\bullet}$ Quick Gel Extraction Kit (Invitrogen, Carlsbad, CA, USA). The purified BoSBP4 gene was ligated into extraction product of pGEX-6p-1 vector using a ClonExpress II One Step Cloning Kit (Vazyme Biotech, Nanjing, China). The pGEX-6p-1 vector sequencing primers were used for sequencing the BoSBP4 gene.

\section{Phylogenetic and bioinformatics analysis}

The amino acid sequence of BoSBP4 was aligned with homologous sequences using MAFFT version 7 (https:// mafft.cbrc.jp/alignment/server/) [32, 33]. Phylogenetic analysis was performed by the maximum likelihood method using MEGA 6.0 software [34].

The numbers of introns and exons were analyzed with the GENSCAN online tool (http://genes.mit.edu/GENSCAN.html) [35]. The predicted amino acid sequence of BoSBP4 was obtained using ExPASY online tool (http:// www.expasy.org/translate/. Open reading frame (ORF) was analyzed by ORF finder (https://www.ncbi.nlm.nih.gov/orffinder/) [36]. Transmembrane (TM) regions, putative glycosylphosphatidy-l-inositol (GPI) anchors and putative signal peptides were predicted by TMHMM 2.0 (http:// www.cbs.dtu.dk/services/TMHMM-2.0/), GPI Prediction Server 3.0 (http://mendel.imp.ac.at/sat/gpi/gpi_server.html) and SignalP 4.1 Server (http://www.cds.dtu.dk/dervices/signalp/), respectively $[37,38]$. The potential subsequence motifs were analyzed with Motif Scan Server (http://mythis.isb-sib.ch/cgi-bin/motif_scan).

The secondary structure of BoSBP4 was predicted using the PROTEAN program of the Lasergene ${ }^{\circ}$ software package in DNAstar in terms of hydrophilicity, flexible region, antigenic index and surface probability. For the prediction of tertiary structure, the amino acid of BoSBP4 was submitted to the I-TASSER server (https://zhanglab.ccmb.med.umich.edu/) [39-41]. There were approximately three steps for prediction. First, structure templates were identified from the PDB library using the local meta threading server (LOMETS) by accumulating high scoring targets for template alignments from nine threading programs $[40,42]$. Ten top threading templates were selected due to higher structure accuracy in conserved regions with the amino acid of BoSBP4. Secondly, based on the above threading template alignments and the five largest structure clusters using SPICKER program, five models were selected $[39,41]$. The one with the best quality among the five selected models was considered as the preliminary tertiary structure of the BoSBP4. Finally, the above selected model was matched with all the structures of proteins in the PDB library and ten top proteins were collected due to their closest structural similarity.

\section{Expression and purification of the recombinant protein}

Because there was a predicted cleavage site between 22 and 23 aa based on the SignalP 4.1 Server prediction website (http://www.cds.dtu.dk/dervices/signalp/), the BoSBP4 gene was truncated and expressed from 70 to $945 \mathrm{bp}$ for the convenience of prokaryotic expression.

The pGEX-6P-1/BoSBP4 truncated recombinant plasmid was confirmed by restriction enzyme digestion and sequencing analysis, then was transformed into $E$. coli BL21 (DE3) strain. Small-scale culture was subjected to 1 $\mathrm{mM}$ isopropyl- $\beta$ - $D$-thiogalactopyranoside (IPTG) induction at $37^{\circ} \mathrm{C}$ for $4 \mathrm{~h}$. The expression form was analyzed by sodium dodecyl sulfate polyacrylamide gel electrophoresis (SDS-PAGE). The recombinant BoSBP4 (rBoSBP4) was expressed as GST-fusion protein and purified using Glutathione Sepharose HP (GE Healthcare, Pittsburgh, PA, USA). In brief, the $E$. coli cells were washed three times with phosphate buffer saline (PBS), and lysed by ultrasonication in binding buffer containing phenylmethylsulfonyl fluoride (PMSF). After centrifugation at $12,000 \times g$ for 15 min at $4{ }^{\circ} \mathrm{C}$, the supernatant was collected, purified with the Glutathione Sepharose HP (GE Healthcare) and stored at $-80{ }^{\circ} \mathrm{C}$ for further use.

\section{Polyclonal antibody preparation}

The rBoSBP4 (100 $\mu \mathrm{g}$ each) in Freund's complete adjuvant (Sigma, San Francisco, CA, USA) was subcutaneously injected into five mice (specific pathogen free). The same antigen administration in Freund's incomplete adjuvant (Sigma) was conducted on the 14, 21 and 28th day. Serum from the five immune mice was collected 14 days after the last immunization and the serum from the healthy mice was collected as control and stored at $-20{ }^{\circ} \mathrm{C}$. The antibody titers of the immune serum were determined by the enzyme-linked immunosorbent assay (ELISA).

\section{SDS-PAGE and Western blot}

To evaluate if $B$. orientalis-infected water buffalo can generate antibodies against SBP4, rBoSBP4 was verified by SDS-PAGE and subsequently blotted on a polyvinylidene fluoride membrane (PVDF, Merck Millipore, Kenny, NJ, USA). The membrane was blocked with 0.05\% Tween-20 in tris buffered saline (TBST) plus 5\% skimmed milk and then probed separately with the serum from $B$. orientalis infected water buffalo (1:400) or normal water buffalo. A secondary antibody (1:2000) of horseradish peroxidase (HRP)-conjugated anti-bovine IgG (Bioss, Beijing, China) was used to identify the bound proteins on the blots.

To identify the native form of SBP4 in B. orientalis merozoite, lysates of $B$. orientalis-infected water buffalo erythrocytes were incubated with the anti-rBoSBP4 mouse serum. Briefly, lysates of $B$. orientalis-infected and uninfected water buffalo erythrocytes were separated on $12 \%$ SDS-PAGE gel, blotted onto a PVDF (Merck Millipore, Kenny, NJ, USA) and probed with the anti-rBoSBP4 mouse serum (1:400) or the serum of naïve mouse or anti-GST tag mouse monoclonal antibody (1:1000, Proteintech Group, 
Chicago, USA) as controls. The membranes were washed with TBST and then incubated with goat anti-mouse IgG/ HRP (1:2000) as secondary antibodies (Bioss). Protein bands on membranes were visualized using the $\mathrm{DAB}$ method (ZSGB-BIO, Beijing, China).

\section{Indirect immunofluorescence assay}

Indirect immunofluorescence assay (IFA) was carried out to determine the cellular localization of BoSBP4. In brief, $B$. orientalis-infected and normal water buffalo erythrocyte smears were fixed in cold 50\% acetone: $50 \%$ methanol (v:v) solution for $30 \mathrm{~min}$ at $-20{ }^{\circ} \mathrm{C}$ and then incubated with the anti-rBoSBP4 mouse serum (1:200), the naïve mouse serum (1:200, negative control) or anti-GST tag mouse monoclonal antibody (1:1000, negative control, Proteintech Group) for $1 \mathrm{~h}$ at $37^{\circ} \mathrm{C}$. After washing three times with the cold PBS, smears were incubated with the second antibody of Alexa-Fluor ${ }^{\circ}$ 594-conjugated goat anti-mouse IgG (1:1000 dilution; Invitrogen) for $1 \mathrm{~h}$ at $37^{\circ} \mathrm{C}$. For nuclear staining, smears were incubated with Hoechst (1:1000 dilution, Invitrogen). Another negative control consisted of the second antibody only without incubation with the primary antibody. Imaging was performed using fluorescence microscopy.

\section{Results}

\section{Characterization of BoSBP4}

BoSBP4 contained an open reading frame of 945 bp without introns, encoding a polypeptide of 314 aa, corresponding to a predicted molecular weight of $37 \mathrm{kDa}$ protein and with an isoelectric point of 4.25 (Fig. 1). The nucleotide sequence of the BoSBP4 gene acquired in this study has been submitted to the GenBank database under the accession number MH429605.

As a signal peptide was cleaved between amino acids 22 and 23, and for the convenience of prokaryotic expression, BoSBP4 gene was truncated and expressed from 70 to 945 bp encoding 291 amino acids. The truncated BoSBP4 gene was expressed as GST-fusion protein in E. coil BL21 (DE3) with a predicted molecular mass of $61 \mathrm{kDa}$. However, the practical molecular weight of the rBoSBP4 in SDS-PAGE was about $70 \mathrm{kDa}$. Finally, the $70 \mathrm{kDa}$ band in SDS-PAGE was identified with MALDI-TOF method, and the result corresponded to the SBP4 of B. bovis, probably due to a fold present in BoSBP4. The rBoSBP4 was extensively expressed in the supernatant and was then purified (Fig. 2).

\section{Phylogenetic and bioinformatics analyses}

The amino acid sequence of BoSBP4 was blasted with the SBP4 sequences in the database. It was found that BoSBP4 showed a query cover of $89 \%$ and an identity of $40 \%$ with SBP4 of B. bovis, and the similarity was mainly located in the $\mathrm{N}$ terminus and the signal peptide site. Additionally, BoSBP4 shared $83 \%$ query cover and only $30 \%$ identity with SBP4 of B. bigemina. The amino acid sequence blast revealed that BoSBP4 was more similar to the SBP4 of $B$. bovis in structure. Amino acid sequence comparison of BoSBP4 with BoSBP3 revealed a query cover of $62 \%$ and an identity of $31 \%$. The similarity mainly ranged from 185 to 290 aa, which may be a conserved domain in the SBP family.

The evolutionary relationships were analyzed according to the amino acid sequences of SBP4 from B. bovis, B. bigemina, Babesia ovata and B. orientalis (Fig. 3). The

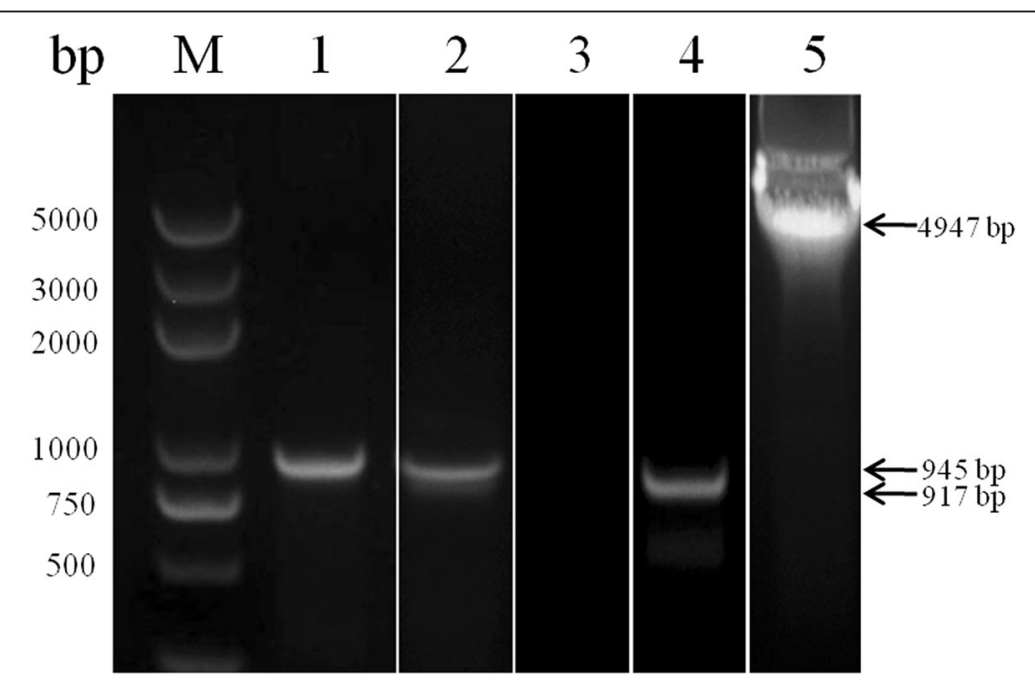

Fig. 1 PCR amplification of the BoSBP4 gene from B. orientalis gDNA and linear pGEX-6P-1 fragment from pGEX-6P-1 plasmid. Lane M: marker; Lane 1: amplificon from gDNA; Lane 2: negative control; Lane 3: amplificon from gDNA for recombinant plasmid construction; Lane 4: amplificon from plasmid of pGEX-6P-1 for recombinant plasmid construction. The corresponding bands are indicated by arrows 


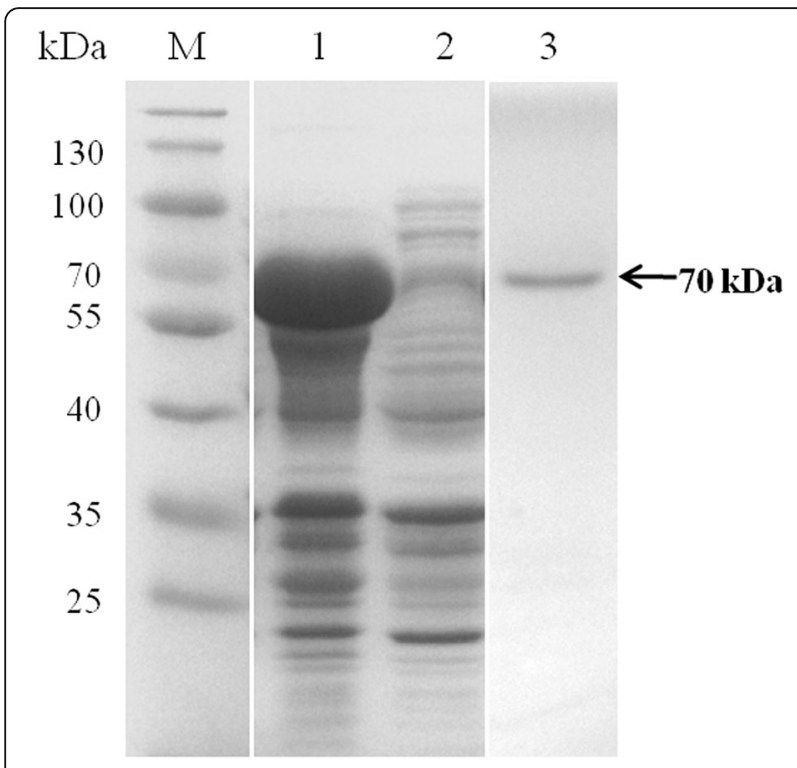

Fig. 2 SDS-PAGE expression and purification of rBoSBP4. Lane M: molecular weight marker; Lane 1: lysate of IPTG induced pGEX-6P-1BoSBP4; Lane 2: lysate of un-induced pGEX-6P-1-BoSBP4; Lane 3: purified product of rBoSBP4. The corresponding bands are indicated by arrows

SBP4 proteins of $B$. orientalis and $B$. bovis were grouped in one clade, and those of B. ovata and B. bigemina were grouped in another clade. However, BoSBP4 and SBP4 of $B$. ovata and B. bigemina were grouped separately. The phylogenetic analysis indicated that BoSBP4 is more close to that of B. bovis, and the SBP4 of B. ovata is more close to that of B. bigemina. In contrast, BoSBP4 was more distantly related to that of $B$. ovata or B. bigemina.
The BoSBP4 gene was predicted to contain one exon and no intron and lack transmembrane regions and GPI anchor sites. The prediction results were consistent with the bioinformatics prediction of the exportome of B. bovis, no transmembrane regions or GPI sites but a signal peptide [43]. The motif scanning of the predicted amino acid sequence of BoSBP4 was performed. It consisted of a N-glycosylation site between 220 and 223 aa, seven casein kinase II phosphorylation sites in the range of 118 to 314 aa, four protein kinase $C$ phosphorylation sites in the range of 5 to 212 aa, two tyrosine kinase phosphorylation sites, one glutamic acid-rich region profile (282-314 aa) and one FAINT region (171-249 aa).

Hydrophilicity, flexible regions, antigenic index and surface probability were predicted based on the corresponding algorithms, including Kyte-Doolittle, KarplusDchulz, Jameson-Wolf and Emini. There were far more hydrophilic regions than hydrophobic regions, with good hydrophilic regions accounting for approximately $75 \%$ of the total number of amino acid residues. Flexible properties were largely related with the amount of helix, turn and coil. The structures of $\alpha$-helix and $\beta$-sheet were undeformable and hard to bind antibodies, which might compromise flexibility. However, turn and coil were similar to the protruding structures, which may facilitate binding with antibodies and serve as potential epitopes. High antigenic regions were uniformly distributed throughout the amino acid sequence and mostly concentrated in the range of 110 to190 aa. The main regions present on the protein surface might be located between 278 and 314 aa. Potential B cell epitopes can be estimated

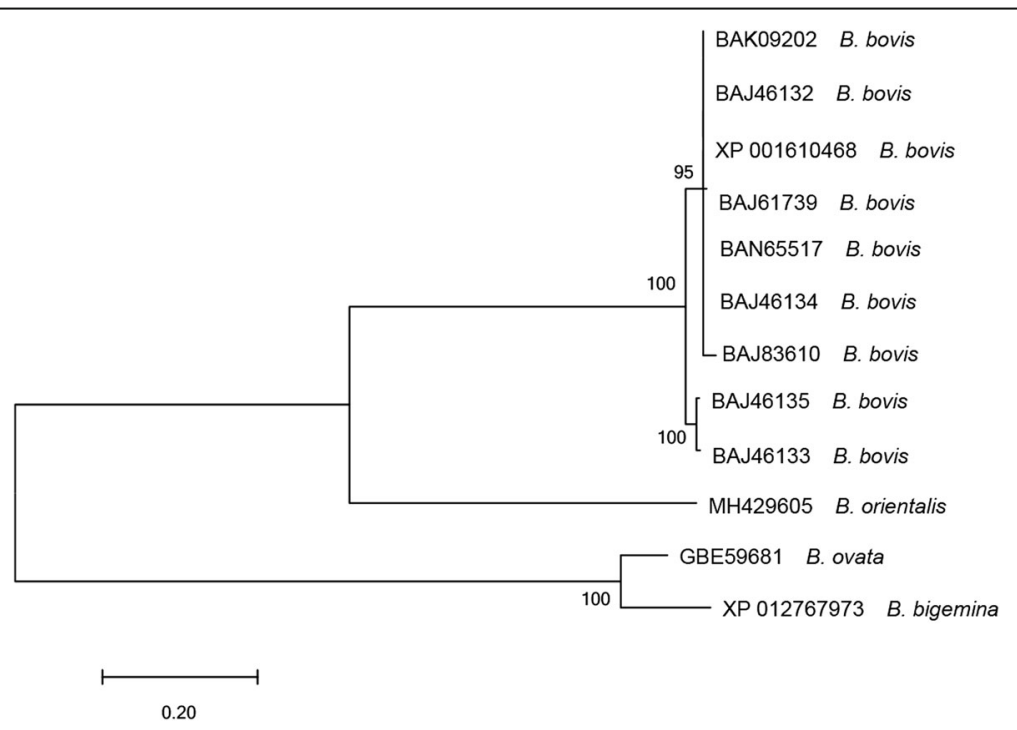

Fig. 3 Molecular phylogenetic analysis of SBP4 amino acid sequences by the maximum likelihood method. The tree is drawn to scale, with branch lengths measured in the number of substitutions per site. The analysis involved 12 amino acid sequences. All positions containing gaps and missing data were eliminated. There were a total of 219 positions in the final dataset. GenBank accession numbers are indicated 
according to these four indices: good hydrophilicity, high flexibility, high accessibility and good antigenicity. Based on comprehensive analysis above, two regions were most likely to be potential B cell epitopes: $107-118$ aa and 278-314 aa.

The three-dimensional structure of BoSBP4 was predicted with the I-TASSER software, with helix, strand and coil distributed throughout the BoSBP4. The schematic illustration of the predicted crystal structure of SBP4 is shown in Fig. 4. Ten proteins were selected due to their similarity in structure to BoSBP4, with the highest score for the anaphase-promoting complex (APC) protein. APC is a ubiquitin ligase that marks target cell cycle proteins for degradation by the $26 \mathrm{~S}$ proteasome [44]. The functions of BoSBP4 were speculated to be most similar to those of the APC, because similar structures may lead to similar functions. The function of APC can serve as a reference for further research on BoSBP4.
Identification of the immunoreactivity and native form of BoSBP4

The immunoreactivity of BoSBP4 was evaluated by incubating rBoSBP4 with the serum from $B$. orientalis-infected water buffalo. A band of about $70 \mathrm{kDa}$ was detected, indicating the generation of antibodies against BoSBP4 in the $B$. orientalis-infected water buffalo (Fig. 5a). Meanwhile, no band was detected in the negative controls. Combined with the predicted secondary structure, the band revealed that BoSBP4 may be a good antigen for the detection of $B$. orientalis.

The expression of BoSBP4 in the merozoite was evaluated by incubation of the lysate of $B$. orientalis-infected erythrocytes with anti-rBoSBP4 serum. A specific band of about $37 \mathrm{kDa}$ was detected, which was consistent with the expected molecular mass of BoSBP4 (Fig. 5b). However, no band was observed in negative controls. This experiment proved that BoSBP4 was actually present in the $B$. orientalis merozoite.

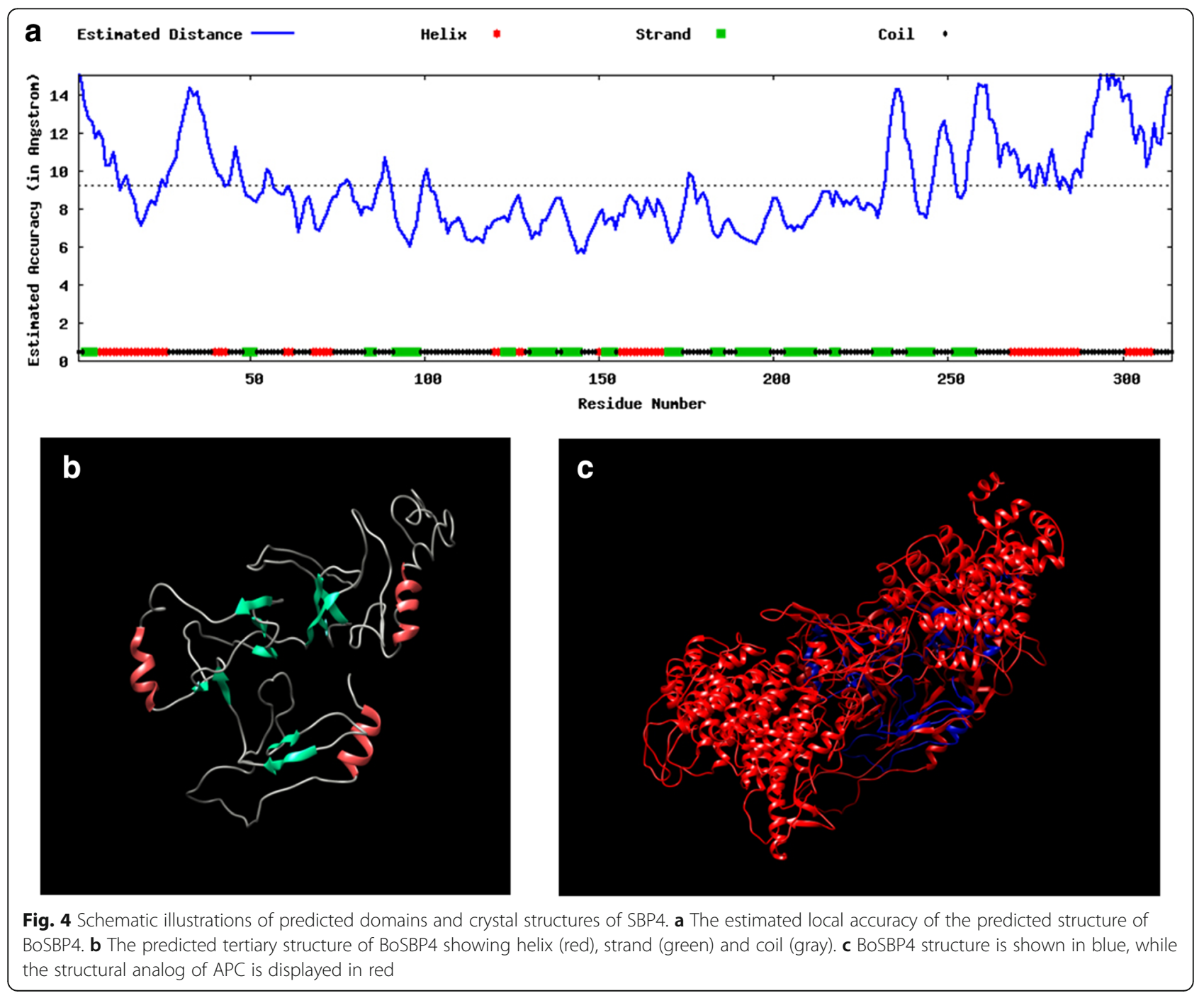




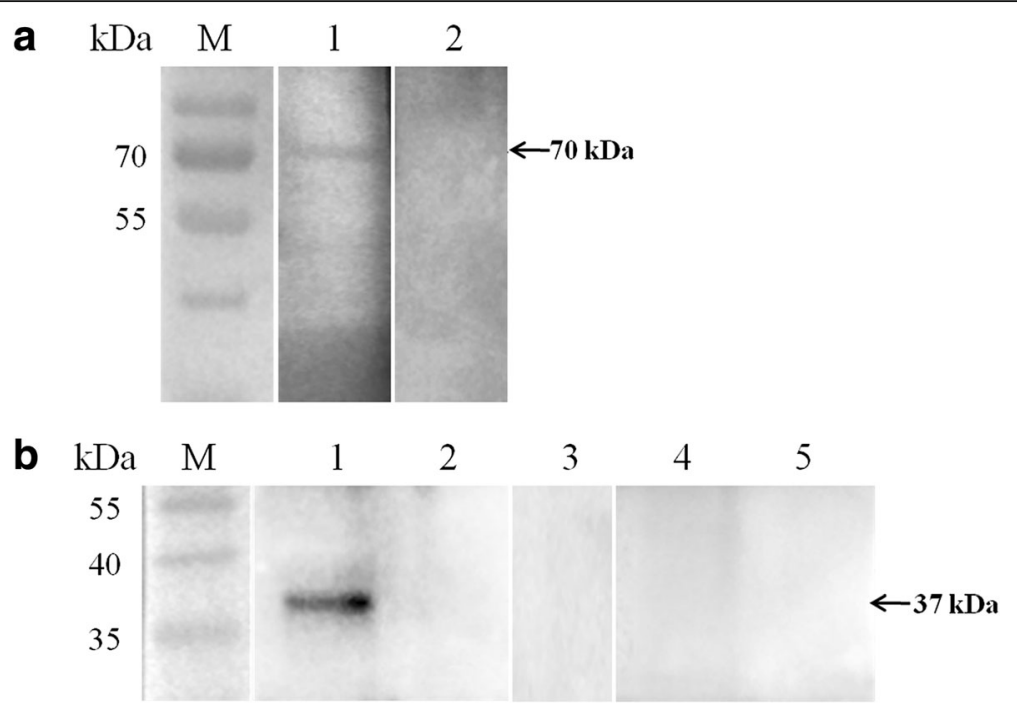

Fig. 5 Western blot identification of immunoreactivity and native form of BoSBP4. a Lane M: molecular weight marker; Lane 1: rBoSBP4 probed with the serum of B. orientalis-infected buffalo; Lane 2: rBoSBP4 probed with the serum of uninfected buffalo. $\mathbf{b}$ Identification of the native form of BoSBP3 in B. orientalis merozoite lysate. Lane M: molecular weight marker; Lane 1: reaction of lysate of B. orientalis-infected erythrocytes with the serum against rBoSBP4; Lane 2: reaction of lysate of uninfected buffalo erythrocytes with the serum against rBoSBP4; Lane 3: lysate of $B$. orientalis-infected buffalo erythrocytes probed with anti-GST tag mouse monoclonal antibody; Lane 4: lysate of $B$. orientalis-infected buffalo erythrocytes probed with the serum of naïve mouse; Lane 5: Iysate of uninfected buffalo erythrocytes probed with the serum of naïve mouse.

The corresponding bands are indicated by arrows

\section{Localization of BoSBP4}

For a better understanding of the properties of BoSBP4, IFA was applied to evaluate the localization of BoSBP4 in merozoite and $\mathrm{iRBC}$. Fluorescence was detected in the intracellular phase. In the iRBC, BoSBP4 was located adjacent to the merozoite nucleus, followed by the diffusion of its fluorescence into the iRBC cytoplasm as puncta-like specks and a gradual increase of the fluorescence (Fig. 6). Meanwhile, no fluorescence could be observed in negative controls.

\section{Discussion}

Due to few studies having been performed so far on $B$. orientalis, its mechanism and properties remain largely unknown. Recently, the mitochondrial and apicoplast genomes of $B$. orientalis were sequenced and analyzed as a supplement to genome analysis (unpublished data), which could facilitate a preliminarily understanding of $B$. orientalis $[3,30,31]$. B. orientalis is an obligate intracellular apicomplexan parasite sharing highly similar characteristics with Plasmodium spp. in many aspects $[8$,

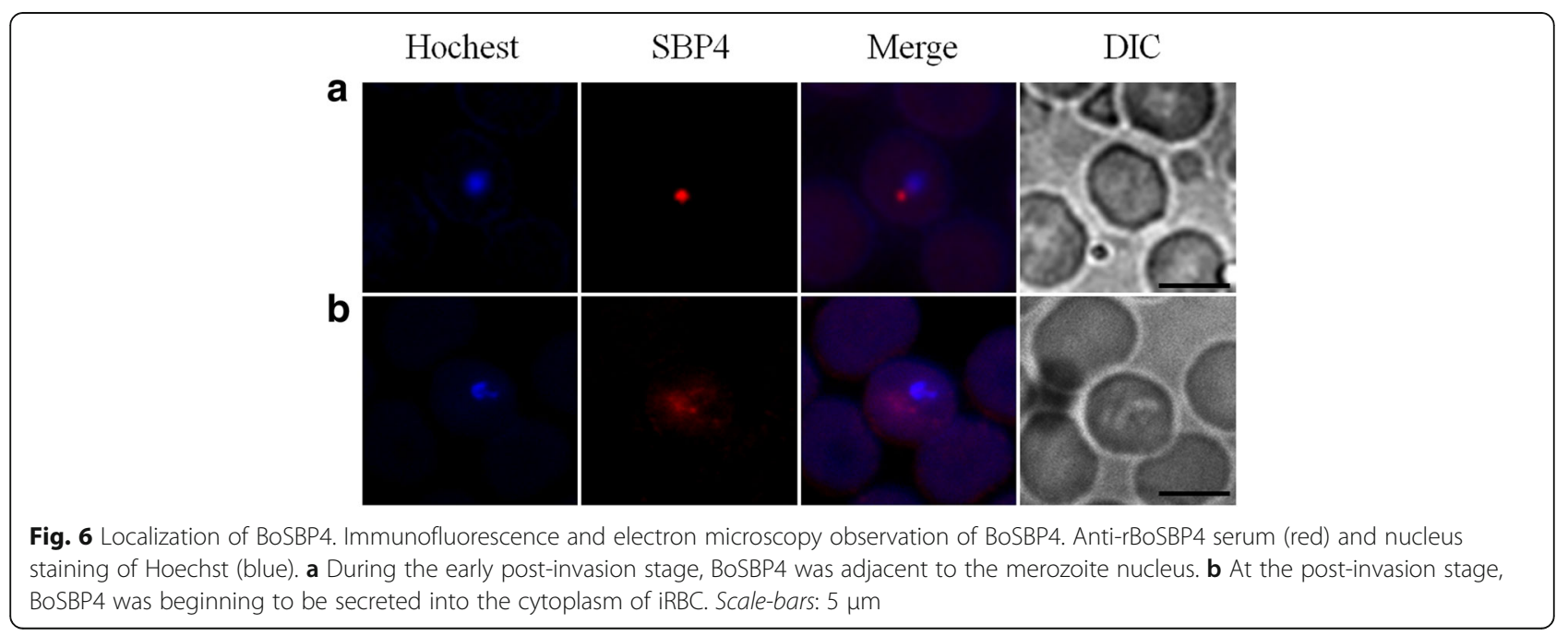


26]. For Plasmodium spp., the first crucial step is to invade the host cell using several proteins secreted by apical organelle complex. For example, apical membrane antigen 1 (AMA1) secreted by micronemes was reported to achieve tight junction formation between parasite and host cell by combining with rhoptry neck proteins $[10$ 12]. The AMA1 of B. orientalis was merely identified and characterized in the merozoite [45], and further research on the AMA1 of $B$. orientalis could refer to the AMA1 in Plasmodium and Toxoplasma gondii that had been extensively studied in molecular functions and related mechanisms $[46,47]$. On the other hand, many differences existed in related proteins and mechanisms between Babesia spp. and Plasmodium spp. For Plasmodium spp., there were four entirely different stages involved in the whole process in the $\mathrm{iRBC}$, including ring stage $(0-24 \mathrm{~h})$, trophozoite stage $(24-36 \mathrm{~h})$, schizogony stage $(36-48 \mathrm{~h})$ and the final merozoite stage [48-50]. However, in Babesia spp., merozoite was the only stage found in the iRBC, leading to far less time in iRBC for Babesia (approximately 6-8 h) than Plasmodium spp. (approximately 36-72 h) $[8,51]$. Therefore, the molecular mediated parasites residing in the host cell might be fewer and more infertile for Babesia than Plasmodium spp. In Plasmodium spp., many proteins were reported to be discharged into the cytoplasm or cytoplasmic face of iRBC to internalize and stabilize the host cell environment for parasite survival and replication [16-18, 5254]. For example, ring-infected erythrocyte surface antigen (RESA) proteins were secreted by dense granules, and then were located to the cytoplasmic face of iRBC through binding the repeat 16 of beta-chain for stabilizing the spectrin tetramer against mechanical and thermal damage [16, 17, 54]. Dense granule antigens (GRAs), secreted by Toxoplasma gondii, were regarded as important targets for the diagnosis and development of vaccines $[55,56]$.

However, there is no consensus sequence in Babesia spp. Instead of dense granules, the spherical body is a unique organelle present in the apical organelle complex of Babesia spp. [19]. So far, four SBPs (SBP1, SBP2, SBP3 and SBP4) have been described for B. bovis in several articles, and for B. bigemina, only the nucleotide sequences of SBP3 and SBP4 were submitted to NCBI [19-21, 24]. Recently, a study has identified for the first time the presence of SBP3 in B. orientalis, which was observed to be secreted into the cytoplasm of iRBC [26].

In this study, the SBP4 gene in B. orientalis was firstly cloned and sequenced, which had an open reading frame of 945 bp with no intron. Meanwhile, BoSBP4 was truncated and expressed for the convenience of prokaryotic expression, because there was a predicted signal peptide cleave site between 22 and 23 aa. For a better understanding of the genetic relationship of SBP4 among different species, the amino acid sequences were blasted with each other. The amino acid homology of BoSBP4 to SBP4 of B. bovis and B. bigemina was $40 \%$ and $30 \%$, respectively, indicating that BoSBP4 was more closely related to $B$. bovis, which is consistent with the phylogenetic analysis between $B$. orientalis and B. bovis in $18 S$ rRNA, AMA1, heat shock protein 90 (HSP90) and HSP70 sequences [3, 45, 57, 58]. BoSBP4 shared $31 \%$ identity with BoSBP3 in the range of 185 to 290 aa, demonstrating a conserved region in the SBP family. For the prediction of motifs in BoSBP4, the phosphorylation sites throughout the BoSBP4 may indicate the extensive modifications in BoSBP4. BoSBP4 also contained one FAINT region from 171 to 249 aa and the motifs of BoSBP4 shared high similarity in structure with SBP4 of $B$. bovis, implying the similarity in essential functions.

Furthermore, in IFA, the fluorescence of BoSBP4 was observed in the intracellular process. Here, BoSBP4 was discharged into the cytoplasm of iRBC as puncta-like specks and gradually increased. The secretion of BoSBP4 into the host cell may be related to the presence of a signal peptide in the $\mathrm{N}$-terminus as a guide. So far, there has been no report about the secretion of other proteins into the iRBC except for SBPs at the late stage of invasion in Babesia spp., suggesting the significant roles of SBPs in the intracellular period. Furthermore, SBPs were identified to be secreted into different regions. SBP1 and SBP2 in B. bovis were characterized to be discharged and concentrated near the inside of the erythrocyte membrane, while SBP3 and SBP4 in B. bovis and BoSBP3 were identified to be secreted throughout the cytoplasm. Moreover, the degree of secretion might vary in SBPs. BoSBP4 was slightly less than BoSBP3 in the fluorescence when discharged into the cytoplasm.

Despite extensive research on SBPs, their mechanisms and functions remain to be further explored [19, 22, 23]. Based on the research of Plasmodium spp., some hypotheses are proposed for Babesia spp. In Plasmodium spp., parasitophorous vacuole (PV) will remain until merozoite egression from the iRBC [59-61]. Additionally, the PV membrane (PVM) is considered to isolate the parasites from the cytoplasm of iRBC to maintain a stable microenvironment (a neutral $\mathrm{PH}$ ) and act as an intermediate between parasites and RBCs [61]. Furthermore, dense granule proteins were reported to participate in host cell internalization [62]. However, for Babesia spp., PVM will disappear with its invasion into the host cell [4]. The special process for Babesia spp. is proposed to be associated with the unique organelle, spherical body, and this needs to be further investigated. Another hypothesis about Babesia spp. is that SBPs are related to the formation of ridges. After invasion into the host cell, parasites will modify RBC in morphology, physiology and biochemistry [53, 63-65]. Some ridge-like protrusion "ridges" will be 
present in the whole RBC membrane surface infected by Babesia spp. [63]. They are similar to the "knobs" present in Plasmodium-infected RBC, and these "knobs" consist of some parasite-derived proteins, such as $P$. falciparum erythrocyte protein 3 (PfEMP3) and KAHRP [53, 63]. For Babesia spp., merely spherical body proteins are known to be released into the iRBC at the late stages. Therefore, SBPs are considered to be related with the formation of "ridges". In addition, the parasitized RBC will adhere to some other cells for immune escape, such as endothelial cells, uninfected RBC and dendritic cells $[64,66]$. The reported related parasite-derived and adhesive protein in $P$. falciparum is $P$. falciparum erythrocyte protein 1 (PfEMP1) that is secreted into the surface of iRBC [52]. In Babesia spp., SBP1 and SBP2 were characterized to localize to the erythrocyte membrane, which may play the same vital roles in adhesion [19, 22]. The aforementioned assumptions about Babesia spp. should be further tested to facilitate a better understanding of the mechanism and functions of SBPs.

Even though the invasion stage is vital to parasites, they will be exposed to the host immune system for a far longer time and with more risks at the post-invasion stage than at the invasion stage. Therefore, the mechanism for the post-invasion stage is worthy of further investigation.

\section{Conclusions}

In this study, a novel spherical body protein in B. orientalis was identified to be secreted into the cytoplasm during its residence in the erythrocyte. BoSBP4 might be functionally important in interaction between the parasite and the host cell to facilitate the parasite survival and multiplication in the erythrocyte. Furthermore, BoSBP4 can be clearly recognized in the serum of $B$. orientalis-infected water buffalo, suggesting the potential application of BoSBP4 to B. orientalis diagnosis. The present study will facilitate the understanding of the SBPs and the development of an effective therapy and prevention against $B$. orientalis infection.

\footnotetext{
Abbreviations

AA: Amino acid; AMA-1: Apical membrane antigen-1; BoSBP4: The SBP4 in Babesia orientalis; CDNA: Complementary DNA; CDS: Sequence coding for amino acids; ELISA: Enzyme-linked immunosorbent assay; gDNA: Genomic DNA; GPI: Glycosylphosphatidy-I-inositol; IFA: Indirect immunofluorescence assay; IP: Isoelectric point; IPTG: Isopropyl- $\beta$-D-thiogalactopyranoside; IRBC: Infected red blood cell; LOMETS: Local meta threading server; ORF: Open reading frame; PBS: Phosphate-buffered saline; PBST: Phosphatebuffered solution Tween-20; PMSF: Phenylmethylsulfonyl fluoride; PV: Parasitophorous vacuole; rBoSBP4: Recombinant BoSBP4; RESA: Ringinfected erythrocyte surface antigen; SBPS: Spherical body proteins; SDSPAGE: Sodium dodecyl sulfate polyacrylamide gel electrophoresis; TBST: Trisbuffered saline Tween-20; TEN: Tris/EDTA/NaCl; TM: Transmembrane
}

\section{Funding}

This study was supported by the National Basic Science Research Program (973 program) of China (grant no. 2015CB150300), the National Key Research and Development Program of China (grant no. 2017YFD0500402 and 2017YFD0501201), the National Natural Science Foundation of China (grant no. 31772729) and the Natural Science Foundation of Hubei Province (grant no. 2017CFA020).

\section{Availability of data and materials}

All data are included as tables and figures within the article. The sequence of BoSBP4 gene generated during the present study is deposited in the GenBank database under the accession number MH429605.

\section{Authors' contributions}

Performed the experiments: JG, YS, LY and JH. Participated in the data analysis: ML, LH, ZN, YS and LY. Helped with the diagnostic assays: PH and ZN. Edited the manuscript: YS, LH and JZ. All authors read and approved the final manuscript.

\section{Ethics approval}

The experimental animals were housed and treated in accordance with the stipulated rules for the regulation of the administration of affair concerning experimental animals of P. R. China. All experiments were performed under the approval of Laboratory Animals Research Centre of Hubei Province and the Ethics Committee of Huazhong Agricultural University (Permit number: HZAUCA-2016-007).

\section{Consent for publication}

Not applicable.

\section{Competing interests}

The authors declare that they have no competing interests.

\section{Publisher's Note}

Springer Nature remains neutral with regard to jurisdictional claims in published maps and institutional affiliations.

\section{Author details}

${ }^{1}$ State Key Laboratory of Agricultural Microbiology, College of Veterinary Medicine, Huazhong Agricultural University, Wuhan 430070, Hubei, China. ${ }^{2}$ Key Laboratory of Animal Epidemical Disease and Infectious Zoonoses, Ministry of Agriculture, Huazhong Agricultural University, Wuhan 430070, Hubei, China. ${ }^{3}$ Key Laboratory of Preventive Veterinary Medicine in Hubei Province, Wuhan 430070, Hubei, China. ${ }^{4}$ College of Life Science, Longyan University \& Fujian, Provincial Key Laboratory for the Prevention and Control of Animal Infectious Diseases and Biotechnology, Longyan 364012, Fujian, China.

Received: 17 May 2018 Accepted: 16 July 2018

Published online: 25 July 2018

\section{References}

1. Goethert HK, Molloy P, Berardi V, Weeks K, Telford SR 3rd. Zoonotic Babesia microti in the northeastern U.S.: evidence for the expansion of a specific parasite lineage. PLoS One. 2018;13:e0193837.

2. Margalit Levi M, Nachum-Biala Y, King R, Baneth G. A survey of Babesia spp. and Hepatozoon spp. in wild canids in Israel. Parasit Vectors. 2018;11:150.

3. He L, Liu Q, Yao B, Zhou Y, Hu M, Fang R, et al. A historical overview of research on Babesia orientalis, a protozoan parasite infecting water buffalo. Front Microbiol. 2017:8:1323.

4. Gohil S, Herrmann S, Gunther S, Cooke BM. Bovine babesiosis in the 21st century: advances in biology and functional genomics. Int J Parasitol. 2013; 43:125-32.

5. He L, Feng HH, Zhang WJ, Zhang QL, Fang R, Wang LX, et al. Occurrence of Theileria and Babesia species in water buffalo (Bubalus babalis, Linnaeus, 1758) in the Hubei Province, South China. Vet Parasitol. 2012;186:490-6.

6. Liu Z, Zhao J, Ma L, Yao B. Babesia orientalis sp. nov. parasitized in buffalo Bubalus bubalis in China (Piroplasmida: Babeslldae). Acta Vet Et Zootech Sin. 1997;28:84-9.

7. Liu Z, Zhao J, Ma L, Yao B. Studies on buffalo babesiosis in Hubei Province, China. Trop Anim Health Prod. 1997;29:33S-6S. 
8. Liu Q, Zhao JL, Zhou YQ, Liu EY, Yao BA, Fu Y. Study on some molecular characterization of Babesia orientalis. Vet Parasitol. 2005;130:191-8.

9. Lobo CA, Rodriguez M, Cursino-Santos JR. Babesia and red cell invasion. Curr Opin Hematol. 2012;19:170-5.

10. Hallee S, Counihan NA, Matthews K, de Koning-Ward TF, Richard D. The malaria parasite Plasmodium falciparum Sortilin is essential for merozoite formation and apical complex biogenesis. Cell Microbiol. 2018;20:e12844.

11. Nasr A, Saleh AM, Eltoum M, Abushouk A, Hamza A, Aljada A, et al. Antibody responses to P. falciparum Apical Membrane Antigen 1 (AMA-1) in relation to haemoglobin $\mathrm{S}(\mathrm{HbS}), \mathrm{HbC}, \mathrm{G} 6 \mathrm{PD}$ and $\mathrm{ABO}$ blood groups among Fulani and Masaleit living in western Sudan. Acta Tropica. 2018;182:115-23.

12. Gaur $D$, Chitnis CE. Molecular interactions and signaling mechanisms during erythrocyte invasion by malaria parasites. Curr Opin Microbiol. 2011;14:422-8.

13. Boyle MJ, Wilson DW, Beeson JG. New approaches to studying Plasmodium falciparum merozoite invasion and insights into invasion biology. Int J Parasitol. 2013;43:1-10

14. Kang JM, Lee J, Moe M, Jun H, Le HG, Kim Tl, et al. Population genetic structure and natural selection of Plasmodium falciparum apical membrane antigen-1 in Myanmar isolates. Malar J. 2018;17:71.

15. Mercier C, Adjogble KD, Daubener W, Delauw MF. Dense granules: are they key organelles to help understand the parasitophorous vacuole of all apicomplexa parasites? Int J Parasitol. 2005;35:829-49.

16. Pei X, Guo X, Coppel R, Bhattacharjee S, Haldar K, Gratzer W, et al. The ringinfected erythrocyte surface antigen (RESA) of Plasmodium falciparum stabilizes spectrin tetramers and suppresses further invasion. Blood. 2007; 110:1036-42.

17. Mills JP, Diez-Silva M, Quinn DJ, Dao M, Lang MJ, Tan KS, et al. Effect of plasmodial RESA protein on deformability of human red blood cells harboring Plasmodium falciparum. Proc Natl Acad Sci USA. 2007;104:9213-7.

18. Morita M, Nagaoka H, Ntege EH, Kanoi BN, Ito D, Nakata T, et al. PV1, a novel Plasmodium falciparum merozoite dense granule protein, interacts with exported protein in infected erythrocytes. Sci Rep. 2018;8:3696.

19. Hines SA, Palmer GH, Brown WC, McElwain TF, Suarez CE, Vidotto O, et al. Genetic and antigenic characterization of Babesia bovis merozoite spherical body protein Bb-1. Mol Biochem Parasitol. 1995;69:149-59.

20. Ruef BJ, Dowling SC, Conley PG, Perryman LE, Brown WC, Jasmer DP, et al. A unique Babesia bovis spherical body protein is conserved among geographic isolates and localizes to the infected erythrocyte membrane. Mol Biochem Parasitol. 2000;105:1-12.

21. Terkawi MA, Huyen NX, Wibowo PE, Seuseu FJ, Aboulaila M, Ueno A, et al. Spherical body protein 4 is a new serological antigen for global detection of Babesia bovis infection in cattle. Clin Vaccine Immunol. 2011;18:337-42.

22. Dowling SC, Perryman LE, Jasmer DP. A Babesia bovis 225-kilodalton spherical-body protein: localization to the cytoplasmic face of infected erythrocytes after merozoite invasion. Infect Immun. 1996;64:2618-26.

23. Tripp CA, Wagner GG, Rice-Ficht AC. Babesia bovis: gene isolation and characterization using a mung bean nuclease-derived expression library. Exp Parasitol. 1989;69:211-25

24. Jasmer DP, Reduker DW, Perryman LE, McGuire TC. A Babesia bovis 225kilodalton protein located on the cytoplasmic side of the erythrocyte membrane has sequence similarity with a region of glycogen phosphorylase. Mol Biochem Parasitol. 1992;52:263-9.

25. Terkawi MA, Seuseu FJ, Eko-Wibowo P, Huyen NX, Minoda Y, AbouLaila M, et al. Secretion of a new spherical body protein of Babesia bovis into the cytoplasm of infected erythrocytes. Mol Biochem Parasitol. 2011;178:40-5.

26. Guo J, Hu J, Sun Y, Yu L, He J, He P, et al. A novel Babesia orientalis 135kilodalton spherical body protein like: identification of its secretion into cytoplasm of infected erythrocytes. Parasit Vectors. 2018;11:205.

27. Dalrymple BP, Peters JM, Goodger BV, Bushell GR, Waltisbuhl DJ, Wright IG. Cloning and characterisation of CDNA clones encoding two Babesia bovis proteins with homologous amino- and carboxy-terminal domains. Mol Biochem Parasitol. 1993;59:181-9.

28. Goodger BV, Waltisbuhl DJ, Commins MA, Wright IG. Babesia bovis: dextran sulphate as an adjuvant for and precipitant of protective immunogens. Int J Parasitol. 1992;22:465-9.

29. Goodger BV, Waltisbuhl DJ, Wright IG, White M. Babesia bovis: analysis of and preliminary vaccination studies with a defined infected erythrocyte membrane binding antigen. Int J Parasitol. 1992;22:533-5.

30. He L, Zhang Y, Zhang QL, Zhang WJ, Feng HH, Khan MK, et al. Mitochondrial genome of Babesia orientalis, apicomplexan parasite of water buffalo (Bubalus babalis Linnaeus, 1758) endemic in China. Parasit Vectors. 2014;7:82.
31. Huang Y, He L, Hu J, He P, He J, Yu L, et al. Characterization and annotation of Babesia orientalis apicoplast genome. Parasit Vectors. 2015;8:543.

32. Katoh K, Rozewicki J, Yamada KD. MAFFT online service: multiple sequence alignment, interactive sequence choice and visualization. Brief Bioinform. 2017;doi: https://doi.org/10.1093/bib/bbx108.

33. Kuraku S, Zmasek CM, Nishimura O, Katoh K. aLeaves facilitates on-demand exploration of metazoan gene family trees on MAFFT sequence alignment server with enhanced interactivity. Nucleic Acids Res. 2013;41:W22-8.

34. Kumar S, Stecher G, Tamura K. MEGA7: Molecular Evolutionary Genetics Analysis Version 7.0 for bigger datasets. Mol Biol Evol. 2016;33:1870-4.

35. Burge C, Karlin S. Prediction of complete gene structures in human genomic DNA. J Mol Biol. 1997;268:78-94.

36. Rombel IT, Sykes KF, Rayner S, Johnston SA. ORF-FINDER: a vector for highthroughput gene identification. Gene. 2002;282:33-41.

37. Bendtsen JD, Nielsen H, von Heijne G, Brunak S. Improved prediction of signal peptides: SignalP 3.0. J Mol Biol. 2004;340:783-95.

38. Krogh A, Larsson B, von Heijne G, Sonnhammer EL. Predicting transmembrane protein topology with a hidden Markov model: application to complete genomes. J Mol Biol. 2001;305:567-80.

39. Yang J, Yan R, Roy A, Xu D, Poisson J, Zhang Y. The I-TASSER Suite: protein structure and function prediction. Nat Methods. 2015;12:7-8.

40. Yang J, Zhang Y. I-TASSER server: new development for protein structure and function predictions. Nucleic Acids Res. 2015:43:W174-81.

41. Zhang Y. I-TASSER: fully automated protein structure prediction in CASP8. Proteins. 2009;77:100-13.

42. Roy A, Yang J, Zhang Y. COFACTOR: an accurate comparative algorithm for structure-based protein function annotation. Nucleic Acids Res. 2012;40:W471-7.

43. Gohil S, Kats LM, Seemann T, Fernandez KM, Siddiqui G, Cooke BM. Bioinformatic prediction of the exportome of Babesia bovis and identification of novel proteins in parasite-infected red blood cells. Int J Parasitol. 2013;43:409-16.

44. Chang L, Zhang Z, Yang J, McLaughlin SH, Barford D. Atomic structure of the APC/C and its mechanism of protein ubiquitination. Nature. 2015;522:450-4.

45. He L, Fan L, Hu J, Miao X, Huang Y, Zhou Y, et al. Characterisation of a Babesia orientalis apical membrane antigen, and comparison of its orthologues among selected apicomplexans. Ticks Tick Borne Dis. 2015;6:290-6.

46. Vetrivel U, Muralikumar S, Mahalakshmi B, Lily Therese K, Madhavan HN, Alameen $\mathrm{M}$, et al. Multilevel precision-based rational design of chemical inhibitors targeting the hydrophobic cleft of Toxoplasma gondii apical membrane antigen 1 (AMA1). Genomics Inform. 2016;142:53-61.

47. Parker ML, Boulanger MJ. An extended surface loop on Toxoplasma gondii apical membrane antigen 1 (AMA1) governs ligand binding selectivity. PLoS One. 2015;10:e0126206.

48. Toenhake CG, Fraschka SA, Vijayabaskar MS, Westhead DR, van Heeringen SJ, Bartfai R. Chromatin accessibility-based characterization of the gene regulatory network underlying Plasmodium falciparum blood-stage development. Cell Host Microbe. 2018;23:557-69.

49. Baum J, Gilberger TW, Frischknecht F, Meissner M. Host-cell invasion by malaria parasites: insights from Plasmodium and Toxoplasma. Trends Parasitol. 2008;24:557-63.

50. Singh $\mathrm{S}$, Chitnis CE. Molecular signaling involved in entry and exit of malaria parasites from host erythrocytes. Cold Spring Harb Perspect Med. 2017;7:6.

51. Yokoyama N, Okamura M, Igarashi I. Erythrocyte invasion by Babesia parasites: current advances in the elucidation of the molecular interactions between the protozoan ligands and host receptors in the invasion stage. Vet Parasitol. 2006;138:22-32.

52. Subramani R, Quadt K, Jeppesen AE, Hempel C, Petersen JE, Hassenkam T, et al. Plasmodium falciparum-infected erythrocyte knob density is linked to the PfEMP1 variant expressed. MBio. 2015;6:e01456-15.

53. Rug M, Prescott SW, Fernandez KM, Cooke BM, Cowman AF. The role of KAHRP domains in knob formation and cytoadherence of $P$ falciparuminfected human erythrocytes. Blood. 2006;108:370-8.

54. Goel S, Muthusamy A, Miao J, Cui L, Salanti A, Winzeler EA, et al. Targeted disruption of a ring-infected erythrocyte surface antigen (RESA)-like export protein gene in Plasmodium falciparum confers stable chondroitin 4-sulfate cytoadherence capacity. J Biol Chem. 2014;289:34408-21.

55. Weeratunga $P$, Herath TUB, Kim TH, Lee HC, Kim JH, Lee BH, et al. Dense Granule Protein-7 (GRA-7) of Toxoplasma gondii inhibits viral replication in vitro and in vivo. J Microbiol. 2017;55:909-17. 
56. Rommereim LM, Bellini V, Fox BA, Petre G, Rak C, Touquet B, et al. Phenotypes associated with knockouts of eight dense granule gene loc (GRA2-9) in virulent Toxoplasma gondii. PLoS One. 2016;11:e0159306.

57. Khan MK, He L, Zhang W, Wang Y, Tao Q, Song Q, et al. Identification of two novel HSP90 proteins in Babesia orientalis: molecular characterization, and computational analyses of their structure, function, antigenicity and inhibitor interaction. Parasit Vectors. 2014;7:293.

58. He L, Liu Q, Quan M, Zhou DN, Zhou YQ, Zhao JL. Molecular cloning and phylogenetic analysis of Babesia orientalis heat shock protein 70 . Vet Parasitol. 2009;162:183-91.

59. Schnider CB, Bausch-Fluck D, Bruhlmann F, Heussler VT, Burda PC. BioID reveals novel proteins of the Plasmodium parasitophorous vacuole membrane. mSphere. 2018;3:1.

60. Khosh-Naucke M, Becker J, Mesen-Ramirez P, Kiani P, Birnbaum J, Frohlke U, et al. Identification of novel parasitophorous vacuole proteins in $P$. falciparum parasites using BiolD. Int J Med Microbiol. 2017;308:1.

61. Hale VL, Watermeyer JM, Hackett F, Vizcay-Barrena G, van Ooij C, Thomas JA, et al. Parasitophorous vacuole poration precedes its rupture and rapid host erythrocyte cytoskeleton collapse in Plasmodium falciparum egress. Proc Natl Acad Sci USA. 2017;114:3439-44.

62. Trager W, Rozario C, Shio H, Williams J, Perkins ME. Transfer of a dense granule protein of Plasmodium falciparum to the membrane of ring stages and isolation of dense granules. Infect Immun. 1992;60:4656-61.

63. Hutchings $\mathrm{CL}$, Li A, Fernandez KM, Fletcher T, Jackson LA, Molloy JB, et al. New insights into the altered adhesive and mechanical properties of red blood cells parasitized by Babesia bovis. Mol Microbiol. 2007;65:1092-105.

64. Cooke BM, Mohandas N, Cowman AF, Coppel RL. Cellular adhesive phenomena in apicomplexan parasites of red blood cells. Vet Parasitol. 2005;132:273-95

65. Molloy JB, Bowles PM, Jorgensen WK, Cooke BM. Babesia bovis: adhesion of parasitized red blood cells to bovine umbilical vein endothelial cells in vitro does not select for virulence. Exp Parasitol. 2003;103:182-4.

66. O'Connor RM, Long JA, Allred DR. Cytoadherence of Babesia bovis-infected erythrocytes to bovine brain capillary endothelial cells provides an in vitro model for sequestration. Infect Immun. 1999;67:3921-8.

Ready to submit your research? Choose BMC and benefit from:

- fast, convenient online submission

- thorough peer review by experienced researchers in your field

- rapid publication on acceptance

- support for research data, including large and complex data types

- gold Open Access which fosters wider collaboration and increased citations

- maximum visibility for your research: over $100 \mathrm{M}$ website views per year

At $\mathrm{BMC}$, research is always in progress.

Learn more biomedcentral.com/submissions 